International Journal of Wireless \& Mobile Networks (IJWMN) Vol. 3, No. 4, August 2011

\title{
A Practical Approach to the Minimization of THE PAgING Costs IN Location MANAGEMENT
}

\author{
E. Martin and R. Bajcsy \\ Department of Electrical Engineering and Computer Science \\ University of California, Berkeley \\ California, USA \\ emartindeecs.berkeley.edu
}

\begin{abstract}
In this paper, we study different mathematical methods to tackle the minimization of the paging costs in Location Management, making use of the probability distribution for the location of mobile terminals. We have covered the classic methods, also considering the paging delay as a constraint, for which case, focus is set mainly on the Simplex method and the Lagrange multiplier method to analyze practical cases, providing useful conclusions on the design of location areas. In particular, making use of the Simplex method for the study of deployments of location areas paged sequentially, the first location areas to be paged should be assigned superior probabilities in order to minimize paging costs, call delivery delays and even computational costs at the time of applying the Simplex method. Regarding the application of Lagrange multipliers, values around 0.1 prove to work efficiently for the typical paging costs optimisation problems and the results obtained show that sequential paging in decreasing order of probability brings large savings in the paging costs with affordable delays: for instance, reduction of $50 \%$ in the costs, for a maximum delay of 3 paging steps.
\end{abstract}

\section{KEYWORDS}

Location Management Costs, Paging Costs, Paging Delay, Mobile Communications Networks.

\section{INTRODUCTION}

The recent growth of users in mobile telecommunications networks has brought the need to solve difficult aspects regarding the mobility of the users and their interaction with the networks [1-2]. The proliferation of new protocols and algorithms aimed at enhancing the network capabilities and providing the user with more and better services has become a constant trend in recent years [3-5]. Nevertheless, there are still open problems concerning Mobility Management that need to be tackled. Special attention must be paid to the efficient use of the scarce radio resources.

Within Mobility Management, Location Management enables the roaming of the user in the coverage area, with the main tasks involved being location update and paging [1-2]. The location update procedure consists of informing the network about every new location the mobile terminal enters, while paging is employed by the network to deliver incoming calls to the user. The signaling messages involved in these two procedures consume a significant proportion of the available radio resources. In order to minimize this signaling burden, the location area concept is used, whereby the mobile terminal will inform the network about a change in its position only when the location area's border has been crossed. The location area's dimension should be optimized according to the call and mobility patterns of the user [3-5].

DOI : 10.5121/ijwmn.2011.3401 
As recent examples, reference [6] uses an ant colony optimization method to minimize the Location Management costs in mobile communication networks, while a differential evolution based algorithm has been employed in [7] to tackle the optimization of Location Management costs. Approaches based on scatter search have also been proposed in $[8,9]$ to optimize costs in network configurations using reporting cells for Location Management. Since user location and mobility patterns can change, dynamic modifications in the layout of location areas can help optimize the Location Management signaling costs (this is becoming a very interesting topic, taking into account that the dynamicity of the user can be easily studied with current state-ofthe-art smart phones [10-12]). For instance, Long Term Evolution (LTE) allows automatic network reconfiguration, which can significantly reduce the overall signaling costs, despite the additional overhead due to the reconfiguration. In this sense, reference [13] focuses on this trade-off inherent to LTE between the total signaling overhead and the reconfiguration cost, applying a genetic algorithm together with local search for its optimization.

It is interesting to note that with the trend to leverage the sensors embedded in smart phones, the information that can be obtained about the users' activities [14-20] and locations [21-22] can be very helpful in assisting network operators optimize the use of the radio spectrum.

In this article, we have studied different mathematical methods to tackle the minimization of the paging costs in Location Management, making use of the probability distribution for the location of the mobile terminal. We have covered the classic methods, also considering the paging delay as a constraint, for which case, focus is set mainly on the Simplex method and on the Lagrange multiplier method to analyze practical cases, providing useful conclusions on the design of location areas. The rest of this article is organized as follows. In Section 2 we present a mathematical definition of the problem, leveraging the probability distributions for the locations of mobile terminals. Section 3 studies the suitability of classic methods for the optimization of this problem. Section 4 considers the introduction of constraints in the minimization of the paging costs, examining the suitability of the Simplex and Lagrange multipliers methods to tackle the problem, showing interesting results useful for the design of cellular networks. Conclusions are drawn in Section 5.

\section{Mathematical Definition of the Problem}

For the analysis of Location Management, a probability distribution for the location of the mobile terminal is commonly assumed. This probability distribution can either be obtained from the analysis of movement models (for instance, random or fluid models) or through measurement reports from the wireless networks. Data from smart phone sensors can also be very useful to assist in solving this problem [10-12, 17, 21-22]. For the particular case of the minimization of the paging costs, the constraint imposed by the possible incurred delay should be considered. Calling $p_{i}$ the probability of the mobile terminal being at a cell $i$, the probability of being in the set of cells that form each location area $j$ can be expressed as follows:

$$
P_{\text {Loc }_{-} \text {Area }} j=\sum_{i=1}^{\text {Nloc }}{ }_{i}^{j} p_{i}
$$

Where $N_{l o c_{-} j}$ is the number of cells within the location area $j$. It must be noticed that this number of cells can be different for every location area.

Classifying the cells in a location area according to their geographical position within it, in such a way that those cells in the periphery are assigned a higher probability of provoking a location 
update due to a movement originated in them than those cells in the inner side, the probability of an update from a particular location area $j$ can be expressed by the following expression:

$$
\begin{aligned}
& P_{\text {Update }_{-} j}=\sum_{i=1}^{I} P_{\text {erif }} i \\
& +\left(h_{i g h}\right)+\sum_{k=1}^{K} I_{n n e r_{-} k} \cdot\left(l_{\text {ow }} k\right)+ \\
& +\sum_{l=1}^{L} I_{n n e r_{-} l} \cdot\left(l_{\text {ow }} l\right)+\ldots+\sum_{q=1}^{Q} I_{n n e r_{-}} q \cdot\left(l_{\text {ow }} q\right)
\end{aligned}
$$

Where $P_{\text {erif }} i$ is the probability of the user being within the $I$ cells in the periphery of the location area, and $I_{n n e r_{-} k}, I_{n n e r_{-} l}, \ldots, I_{n n e r_{-} q}$ are the probabilities of the user being in the different inner groups of respectively $K, L, \ldots Q$ cells, organized according to their proximity to the periphery, and with probabilities of triggering an update $l_{o w_{-} k}, l_{o w_{-} l}, \ldots, l_{o w_{-}} q$ respectively. Consequently, the location update costs for a deployment in terms of probabilities, will be proportional to the summation of the probabilities of an update from the $J$ location areas being considered:

$$
\text { Cost } t_{\text {updating }}=\sum_{j=1}^{J} P_{\text {Update }_{-} j} \cdot \text { Cost }_{-} \text {each } \text { update }_{\text {und }}
$$

Therefore, by means of (3), the minimization of the location update costs could be tackled from a mathematical point of view. In this paper we will focus on the minimization of the paging costs. Considering sequential paging of location areas, ordered according to the probabilities of the mobile user being within them, and assuming that all the cells inside a location area are paged simultaneously, the number of cells paged to find a mobile terminal is:

$$
\text { Number_paged }{ }_{-} j=\sum_{r=1}^{j} N_{l o c_{-} r}
$$

Where $j$ represents the order of the location area where the mobile terminal stays. Consequently, in terms of probabilities, the number of cells the network has to page in order to find the mobile terminal, in other words, the cost of paging for that mobile terminal is:

$$
\text { Cost }_{\text {paging }}=\sum_{j=1}^{N}\left(\text { Number }_{-} \text {paged }_{-} j\right) \cdot\left({\text { P } \text { Loc }_{-} \text {Area }_{-} j}\right)
$$

Where $N$ is the total number of location areas. In general, the number of cells in every location area has been previously established by the designer, and the variables are the probabilities of every location area, which in turn are the summation of the probabilities of the cells inside. Therefore, from a mathematical point of view, the paging cost is a function of $N$ variables, where $N$ is determined by the designer. Next, different optimisation methods will be applied for the optimization of the paging costs.

\section{Classic Methods}

\subsection{Classical Theory of Maxima and Minima}

Problems not too complex and not involving more than a few variables can be easily solved by classical optimisation techniques. The theory of maxima or minima and their necessary and sufficient conditions for a single variable function can be applied to functions of several variables like paging costs, with certain particularities such as the introduction of the Hessian 
matrix. If the cost of paging takes a minimum for certain values of the variables, the first partial derivatives of the function with respect to each of the $N$ variables will vanish for those values of the variables. And the sufficient condition for the function to have a minimum is that the Hessian matrix be positive definite. However, this method requires high numerical computation to determine whether the Hessian matrix is positive definite or not, which makes it not worth performing [23]. Moreover, if the paging cost takes the expression of a linear combination of the probabilities of the location areas multiplied by scalars, equating the first derivatives to zero will not determine any value of the variables. Consequently, whenever the number of cells in every location area is independent from the probabilities of the mobile terminal being inside a particular location area, the classical theory of maxima and minima should not be applied. Nevertheless, carefully analysing both quantities, it could be possible to obtain a relationship between them. An attempt to do so, would be, for instance, considering the total number of cells in the design as a summation of the probabilities of each location area divided by a proportionality constant which measures the preference of the designer to give higher priorities to first locations.

In this sense, considering for example $C$ cells within which the mobile terminal is expected to be roaming at least $T \%$ of the time, and organizing them in $N$ location areas ordered according to their priorities, a proportionality constant could be assigned to each location area in the following way: each location area is given different priority values, for instance $\alpha_{i} \cdot P$ to the location area with order $i, \alpha_{i+1} \cdot P$ to the location area with order $i+1$, etc., where the different $\alpha_{i} \cdot P$ values measure the priorities of the location areas according to the designer's criteria, and they will have to fulfil the following condition: $\alpha_{1} \cdot P+\ldots+\alpha_{i} \cdot P+\alpha_{i+1} \cdot P+\ldots+\alpha_{N} \cdot P=T$. Taking these values into account, the number of cells inside each location area could be expressed as:

$$
\begin{aligned}
& N_{l o c_{-} 1}=\frac{P_{\text {Loc }_{-} \text {Area }_{-} 1}}{\alpha_{1} \cdot P} \\
& N_{l o c_{-} 2}=\frac{P_{\text {Loc }_{-} \text {Area }} 2}{\alpha_{2} \cdot P} \\
& \vdots \\
& N_{l o c_{-} N}=\frac{P_{L c_{-} \text {Area }} 4}{\alpha_{N} \cdot P}
\end{aligned}
$$

And the relationship between all of them is give by the following expression:

$$
\frac{P_{\text {Loc }_{-} \text {Area }} 1}{\alpha_{1} \cdot P}+\frac{P_{\text {Loc }_{-} \text {Area }}-2}{\alpha_{2} \cdot P}+\ldots+\frac{P_{\text {Loc }_{-} \text {Area }_{-} N}}{\alpha_{N} \cdot P}=C
$$

Substituting these values in the expression of the paging costs, and equating the first derivatives of that expression with respect to each one of the probabilities to zero, a linear system of $N$ equations with $N$ variables is obtained. However, no physical meaning for the probabilities is inferred if the system is solved, as the solutions for the probabilities would be zero. Consequently, further analysis could be carried out in order to obtain some relationship between the introduced proportionality constants and the location areas probabilities, in order to obtain a 
physical meaning for the solution of equating the first derivatives of the paging costs to zero. However, the complication of the model may not be worth it, considering the trade-off between the accuracy gained in the solution and the complexity added in the calculations.

\subsection{Minimum Paging Costs for the Upper and Lower Bounds of the Variables}

If the values of the variables are restricted in a certain domain, considering the minimum occurring when one or more of the variables are at their upper or lower bounds, there are $2^{N}$ possible different combinations of the variables. For example, with 4 location areas, for $N_{l o c_{-} 1}=5, N_{l o c_{-} 2}=4, N_{l o c_{-} 3}=3$ and $N_{l o c_{-} 4}=5$, the expression for the paging costs is:

$$
\text { Cost }_{\text {paging }}=5 \cdot\left(P_{\text {Loc }_{-} \text {Area }} 1\right)+9 \cdot\left(P_{\text {Loc }_{-} \text {Area }} 2\right)+12 \cdot\left(P_{\text {Loc }_{-} \text {Area }}-3\right)+17 \cdot\left(P_{\text {Loc }_{-} \text {Area }} 4\right)
$$

Considering the probabilities of the location areas to be restricted to the following ranges: $0.5 \leq P_{\text {Loc }_{-} \text {Area }} 1 \leq 0.8, \quad 0.1 \leq P_{\text {Loc }_{-} \text {Area }_{-} 2} \leq 0.3, \quad 0.01 \leq P_{\text {Loc }_{-} \text {Area }} 3 \leq 0.1$, $0.01 \leq P_{\mathrm{Loc}_{-} \text {Area }} 4 \leq 0.1$, computing the function at each one of the 16 possible combinations of the variables, the minimum takes place for the combination of the minimum bound values of every variable, which seemed obvious from the beginning. However, some kind of mathematical restriction should be imposed, so that the different combinations of probabilities add up a minimum threshold. Otherwise the minimum obtained could imply a big uncertainty in terms of finding the mobile user inside the selected areas. The next step should be obtaining the roots of the first derivatives of the paging costs, checking if any of the roots lies in the predetermined range for the probabilities, and the optimum point would be the one that makes the costs take the lowest value, chosen between the obtained set of roots for the first derivatives and the set of $2^{N}$ possible different combinations that the probabilities may take considering their upper or lower bounds. In this case, there is no point in working with the first derivatives when the paging cost is a linear function of the probabilities multiplied by scalars. However, it can be noticed that the large number of first derivatives for which the roots should have to be calculated in case of a different type of function is an important drawback for this method [24, 25]. Even more, another computational drawback of this method is the fact that $2^{N}$ combinations of conditions have to be tested, and for large numbers of variables, the computational burden can be very important.

\section{Minimization of Paging Costs Subject to Constraints}

Paging costs optimisation techniques usually bring costs savings at the expense of some delay. Considering all the cells within a location area paged simultaneously, and location areas paged sequentially according to an established order, the delay units incurred to find a mobile terminal equal the number of location areas paged. In terms of probabilities, the mean delay incurred for the case of $N$ location areas is:

Del $=\sum_{j=1}^{N} j \cdot P_{\text {Loc }_{-} \text {Area }_{-} j}$

This delay represents a constraint for the paging costs function, and next we will study the suitability of the application of the Simplex and Lagrange multiplier methods to tackle the minimization of the paging costs subject to a constraint.

\subsection{Basic Introduction to Linear Programming}

Linear Programming is a very common optimisation technique for problems with the following pattern: 


$$
\begin{aligned}
& \text { Optimize } \quad f=\sum_{j=1}^{N} s_{j} \cdot x_{j} \quad \text { subject to : } \quad \sum_{j=1}^{N} a_{i j} \cdot x_{j}=c_{i} \\
& \text { with } i=1 . . M \quad \text { and } \quad x_{j} \geq 0
\end{aligned}
$$

In this expression, all the coefficients are real scalars and the function to optimise is a linear form in the variables to be solved, $x_{j}$. Also the constraints are linear equalities or inequalities that will eventually be transformed into equalities. There are various methods to solve linear programming problems, such as the Transportation Technique, the Modi method, the Ratio Analysis, the Simplex method, and the Index method [26]. Amongst them, the Simplex method can be considered as the basic linear programming method, as all the other methods originated from it [26].

\subsubsection{Simplex Method}

The Simplex method was developed by G. Dantzig to be applied to linear programming problems expressed in their canonical form as:

Minimize $s \cdot x=m$, subject to $A \cdot x=c$, where $x$ represents the column vector of variables to be solved, s represents the row vector of the coefficients multiplying the variables in the formula to be minimized, $A$ represents the matrix of coefficients for the constraints equations, and $c$ represents the column vector of independent terms for the constraints equations. Working with the minimization of paging costs, $x$ would stand for the probabilities of the mobile terminal staying within the location areas, and $s$ would stand for the corresponding summations of the numbers of cells inside the location areas. The $A$ matrix would be obtained from the set of constraints affecting the probabilities, such as imposing certain thresholds for the probabilities, or constraining them by an expected maximum value of the delay to deliver a call.

The method consists of steadily obtaining better feasible solutions making use of suitable algebraic operations with the coefficients of the problem. For example, given 4 location areas, with the following number of cells for each one of them: 5 cells for the first, 4 cells for the second, 3 cells for the third, and 6 cells for the fourth, the paging costs would be expressed as follows:

$$
\text { Cost }_{\text {paging }}=5 \cdot P_{\text {Loc }_{-} \text {Area }_{-} 1}+9 \cdot P_{\text {Loc }_{-} \text {Area }_{-} 2}+12 \cdot P_{\text {Loc }_{-} \text {Area }} 3+18 \cdot P_{\text {Loc }_{-} \text {Area }_{-} 4}
$$

In order to minimize these costs, considering some constraints, for example a first constraint imposing a certainty of at least $95 \%$ of the time for the mobile terminal being within the 4 location areas being studied, a second constraint expressing a restriction in terms of maximum allowed delay value of 1.5 time units to deliver a call, and 4 more constraints imposing some minimum thresholds of probabilities for the different location areas, the constraints would take the following form:

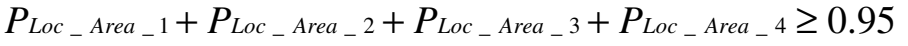

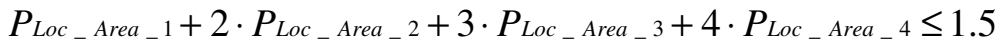

$$
\begin{aligned}
& P_{\text {Loc }_{-} \text {Area }} 1 \geq 0.5 \\
& P_{\text {Loc }} \text { Area } 22 \geq 0.3 \\
& P_{\text {Loc }_{-} \text {Area }} 3 \mathrm{3} \geq 0.1 \\
& P_{\text {Loc }_{-} \text {Area }_{-} 4} \geq 0.01
\end{aligned}
$$


The Simplex method transforms the $\leq$ inequalities into equalities by means of adding nonnegative slack variables. In this sense, the second inequality constraint in (12) is transformed into:

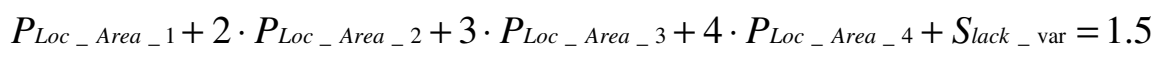

The $\geq$ inequalities are transformed into equalities by means of subtracting slack variables and adding artificial variables, also non-negative. For instance, the first inequality in (12) is transformed in the following way:

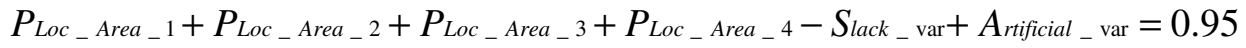

Next, the so-called Simplex Tableaux are used to work with the coefficients of the problem, and iteratively obtain better solutions. For the previous data, transforming the inequalities into equalities, the problem takes the following form:

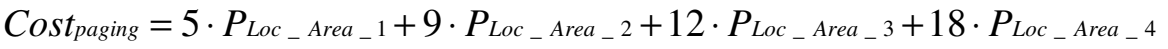

$$
\begin{aligned}
& P_{\text {Loc }_{-} \text {Area }} 1+P_{\text {Loc }_{-} \text {Area }} 2+P_{\text {Loc }_{-} \text {Area }_{-} 3}+P_{\text {Loc }_{-} \text {Area }_{-} 4}-S_{\text {lack }} \text { var } 1+A_{\text {rtificial }} \text { var } 1=0.95
\end{aligned}
$$

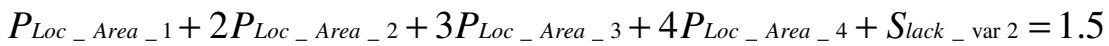

$$
\begin{aligned}
& P_{\text {Loc_Area_ }_{1}}-S_{\text {lack }} \text { var } 3+A_{\text {rtificial }} \text { var }_{2}=0.5 \\
& P_{\text {Loc_Area_2 }}{ }_{-} \text {Slack_var } 4_{4}+A_{\text {rtificial }} \text { var } 3_{3}=0.3 \\
& P_{\text {Loc }_{-} \text {Area_ }}{ }_{3}-S_{\text {lack_var } 5}+A_{\text {rtificial }} \text { var }_{4}=0.1
\end{aligned}
$$

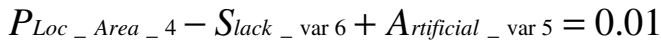

Then, calling the different $P_{\text {Loc }_{-} \text {Area }} i$ as $x_{i}$, and manipulating the equations to work with the Tableaux, the problem can be expressed as follows:

$$
\begin{aligned}
& \text { Minimize } m=5 x_{1}+9 x_{2}+12 x_{3}+18 x_{4} \quad \text { Subject to : } \\
& x_{1}+x_{2}+x_{3}+x_{4}-x_{5}+x_{11} \quad=0.95 \\
& \begin{array}{lll}
x_{1}+2 x_{2}+3 x_{3}+4 x_{4}+x_{6} & =1.5
\end{array} \\
& \begin{array}{llll}
x_{1} & -x_{7} & +x_{12} & =0.5
\end{array}
\end{aligned}
$$

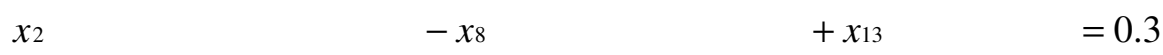

$$
\begin{aligned}
& \begin{array}{llll}
x_{3} & -x_{9} & +x_{14} & =0.1
\end{array} \\
& \begin{array}{llll}
x_{4} & -x_{10} & +x_{15} & =0.01
\end{array}
\end{aligned}
$$

For the typical costs minimization problems that can be solved through the Simplex method, considering the number of constraints imposed lower than 50, it has been reported that the number of iterations required is usually less than 1.5 times the number of constraints [27]. Different researchers analyzing the speed of convergence of the Simplex method have drawn the same previous conclusion about the number of iterations required to solve a typical problem [28]. And although some problems have been reported to require an amazingly large amount of iterations [29], the form of those problems is rarely taken in typical paging costs minimization cases. 
Implementing the algorithm in MATLAB, and analyzing different possible cases for 4 location areas, varying the constraints for the ranges of the probabilities of the location areas and the maximum value for the call delivery delay, interesting results are obtained, which we summarize in Table 1:

Table 1. Summary of results from the application of Simplex method to optimise paging costs.

\begin{tabular}{|l|l|l|l|l|l|l|l|l|c|c|c|}
\hline \multicolumn{3}{|c|}{$\begin{array}{l}\text { Imposed Constraints to } \\
\text { Probabilities of Location Areas }\end{array}$} & \multicolumn{2}{c|}{$\begin{array}{c}\text { Optimum Solution for } \\
\text { Probabilities of Location } \\
\text { Areas }\end{array}$} & $\begin{array}{c}\text { No. } \\
\text { Tables } \\
\text { needed }\end{array}$ & $\begin{array}{c}\text { Paging } \\
\text { Costs } \\
\text { solution }\end{array}$ & $\begin{array}{c}\text { Call } \\
\text { Delivery } \\
\text { Delay }\end{array}$ \\
\cline { 1 - 1 } L.A. 1 & L.A.2 & L.A. 3 & L.A.4 & L.A.1 & L.A.2 & L.A.3 & L.A.4 & & & \\
\hline$\geq 0.2$ & $\geq 0.2$ & $\geq 0.2$ & $\geq 0.2$ & 0.35 & 0.2 & 0.2 & 0.2 & 9 & 9.55 & 2.15 \\
\hline$\geq 0.15$ & $\geq 0.15$ & $\geq 0.15$ & $\geq 0.15$ & 0.5 & 0.15 & 0.15 & 0.15 & 9 & 8.35 & 1.85 \\
\hline$\geq 0.1$ & $\geq 0.1$ & $\geq 0.1$ & $\geq 0.1$ & 0.65 & 0.1 & 0.1 & 0.1 & 9 & 7.15 & 1.55 \\
\hline$\geq 0.05$ & $\geq 0.05$ & $\geq 0.05$ & $\geq 0.05$ & 0.8 & 0.05 & 0.05 & 0.05 & 8 & 5.95 & 1.25 \\
\hline$\geq 0.01$ & $\geq 0.01$ & $\geq 0.01$ & $\geq 0.01$ & 0.92 & 0.01 & 0.01 & 0.01 & 8 & 4.99 & 1.08 \\
\hline$\geq 0.005$ & $\geq 0.005$ & $\geq 0.005$ & $\geq 0.005$ & 0.935 & 0.005 & 0.005 & 0.005 & 8 & 4.87 & 0.98 \\
\hline$\geq 0.001$ & $\geq 0.001$ & $\geq 0.001$ & $\geq 0.001$ & 0.947 & 0.001 & 0.001 & 0.001 & 8 & 4.774 & 0.956 \\
\hline$\geq 0.001$ & $\geq 0.1$ & $\geq 0.001$ & $\geq 0.001$ & 0.848 & 0.1 & 0.001 & 0.001 & 8 & 5.17 & 1.055 \\
\hline$\geq 0.001$ & $\geq 0.001$ & $\geq 0.1$ & $\geq 0.001$ & 0.848 & 0.001 & 0.1 & 0.001 & 8 & 5.467 & 1.154 \\
\hline$\geq 0.001$ & $\geq 0.001$ & $\geq 0.001$ & $\geq 0.1$ & 0.848 & 0.001 & 0.001 & 0.1 & 8 & 6.061 & 1.253 \\
\hline$\geq 0.001$ & $\geq 0.2$ & $\geq 0.001$ & $\geq 0.001$ & 0.748 & 0.2 & 0.001 & 0.001 & 8 & 5.57 & 1.155 \\
\hline$\geq 0.001$ & $\geq 0.001$ & $\geq 0.2$ & $\geq 0.001$ & 0.748 & 0.001 & 0.2 & 0.001 & 8 & 6.167 & 1.354 \\
\hline$\geq 0.001$ & $\geq 0.001$ & $\geq 0.001$ & $\geq 0.2$ & 0.748 & 0.001 & 0.001 & 0.2 & 9 & 7.361 & 1.553 \\
\hline$\geq 0.001$ & $\geq 0.3$ & $\geq 0.001$ & $\geq 0.001$ & 0.648 & 0.3 & 0.001 & 0.001 & 8 & 5.97 & 1.255 \\
\hline$\geq 0.001$ & $\geq 0.001$ & $\geq 0.3$ & $\geq 0.001$ & 0.648 & 0.001 & 0.3 & 0.001 & 10 & 6.867 & 1.554 \\
\hline$\geq 0.001$ & $\geq 0.001$ & $\geq 0.001$ & $\geq 0.3$ & 0.648 & 0.001 & 0.001 & 0.3 & 9 & 8.661 & 1.853 \\
\hline$\geq 0.001$ & $\geq 0.5$ & $\geq 0.001$ & $\geq 0.001$ & 0.448 & 0.5 & 0.001 & 0.001 & 8 & 6.77 & 1.495 \\
\hline$\geq 0.001$ & $\geq 0.001$ & $\geq 0.5$ & $\geq 0.001$ & 0.448 & 0.001 & 0.5 & 0.001 & 8 & 8.267 & 1.954 \\
\hline$\geq 0.001$ & $\geq 0.001$ & $\geq 0.001$ & $\geq 0.5$ & 0.448 & 0.001 & 0.001 & 0.5 & 9 & 11.261 & 2.453 \\
\hline$\geq 0.001$ & $\geq 0.7$ & $\geq 0.001$ & $\geq 0.001$ & 0.248 & 0.7 & 0.001 & 0.001 & 8 & 7.57 & 1.655 \\
\hline$\geq 0.001$ & $\geq 0.001$ & $\geq 0.7$ & $\geq 0.001$ & 0.248 & 0.001 & 0.7 & 0.001 & 8 & 9.667 & 2.354 \\
\hline$\geq 0.001$ & $\geq 0.001$ & $\geq 0.001$ & $\geq 0.7$ & 0.248 & 0.001 & 0.001 & 0.7 & 8 & 13.861 & 3.053 \\
\hline
\end{tabular}

Each row in Table 1 represents a different case, for which the corresponding numbers of Tableaux have been generated. Examining the results from Table 1, it can be noticed that when all the probabilities for the location areas are allowed to range freely within the same interval, 
As a summary from these results, the following conclusion is reached: whenever a location area different from the first one is selected to endure a lower bound value constraint for its probability higher than the rest of the location areas, which are allowed to range freely above a minimum common threshold of probability constraint, then, in order to minimize the paging costs and delay constraint of the design, the different lower bound value should be as low as possible, and be allocated to a location area with the lowest order number, if not the first location area. In other words: if a location area different from the first one to be paged is imposed a lower bound constraint for its probability higher than the rest of location areas, the higher that lower bound constraint is, the larger the paging costs and call delivery delays are. And for the same lower bound value, the higher the order number of the location area for which the different constraint is imposed, the larger the paging costs and call delivery delays will be.

\subsection{Lagrange Multipliers}

Another technique to minimize a function subject to a constraint, is the Lagrange multiplier method, which consists of introducing an auxiliary quantity called the Lagrange multiplier, $\lambda$, constructing what is known as the Lagrangian function:

$$
F(x, \lambda)=f(x)+\lambda[b-g(x)]
$$

Where $f(x)$ is the function to optimise and $g(x)=b$ is the constraint equation. In order to obtain necessary conditions for the existence of a solution to the problem, this new function should be differentiated with respect to each one of the variables and $\lambda$, and equated to zero. In case the constraints are expressed in terms of inequalities, an extension of the Lagrange multipliers can be applied, converting the inequalities into equalities by means of the introduction of auxiliary quantities; for example, $g(x) \leq b$ is turned into $g(x)+s=b$, and the Lagrangian function is now:

$$
F(x, \lambda)=f(x)+\lambda[b-s-g(x)]
$$

For the practical case of optimizing the paging costs subject to the constrain of the call delivery delay, the Lagrange multiplier method takes the following expression:

$$
\begin{aligned}
& F=\text { Cost }_{\text {paging }}+\lambda \cdot(b-\mathrm{Del})= \\
& =\sum_{j=1}^{N}\left(\text { Number }_{-} \text {paged }_{-} j\right) \cdot\left(P_{\text {Loc }_{-} \text {Area }} j\right)+\lambda \cdot\left[b-\sum_{j=1}^{N} j \cdot\left(P_{\text {Loc }_{-} \text {Area }_{-} j}\right)\right]
\end{aligned}
$$

Where $N$ is the number of location areas considered. A way to supply an overbound on the minimum paging costs and call delivery delay for any location probability distribution is to work with the uniform distribution, which will act as a worst case, due to the fact of having the maximum stochastic order [30]. Furthermore, taking into account that continuous solutions underbound discrete solutions [30], considering a continuous uniform distribution defined on [0, I], the mean call delivery delay can be expressed as [30]:

$$
\text { Del }=\sum_{j=1}^{N} j\left[\frac{1}{N}+\frac{\lambda}{2 I}(N+1)-j \frac{\lambda}{I}\right]
$$

And the paging costs for the same distribution can be calculated as in [30]: 


$$
\text { Cost }_{\text {paging }}=\sum_{j=1}^{N} j\left[\frac{I}{N}+\frac{\lambda}{2}(N-j)\right] \cdot\left[\frac{1}{N}+\frac{\lambda}{2 I}(N+1)-j \frac{\lambda}{I}\right]
$$

For low values of the Lagrange multiplier, the behaviour of the solution for the paging costs and delay is coherent with the expected physical solution (paging costs decrease at the expense of a delay rise), but as the maximum number of paging steps grows, the mathematical nature of the method makes the obtained solution lose physical meaning (the obtained solution for the delay really starts decreasing after a certain figure for the maximum number of paging steps, and the paging costs start picking up at that exact point). As the maximum number of paging steps continues increasing, the delay keeps on falling, even reaching negative values, totally meaningless in a physical sense. This trend is more emphasized with higher the value of the Lagrange multiplier. In Table 2, a numerical summary of the described behaviour is presented.

Table 2. Results from the application of the Lagrange multiplier.

\begin{tabular}{|c|c|c|}
\hline \multirow{2}{*}{$\begin{array}{c}\text { Lagrange } \\
\text { Multiplier }\end{array}$} & Maximum number of paging steps before call delivery delays start having: \\
\cline { 2 - 3 } & Abnormal behaviour & Negative values \\
\hline 0.01 & 374 & 648 \\
\hline 0.05 & 45 & 246 \\
\hline 0.1 & 32 & 173 \\
\hline 0.2 & 12 & 32 \\
\hline 0.7 & 7 & 11 \\
\hline 1.5 & 5 & 8 \\
\hline
\end{tabular}

This is shown graphically in Figures 1 and 2:

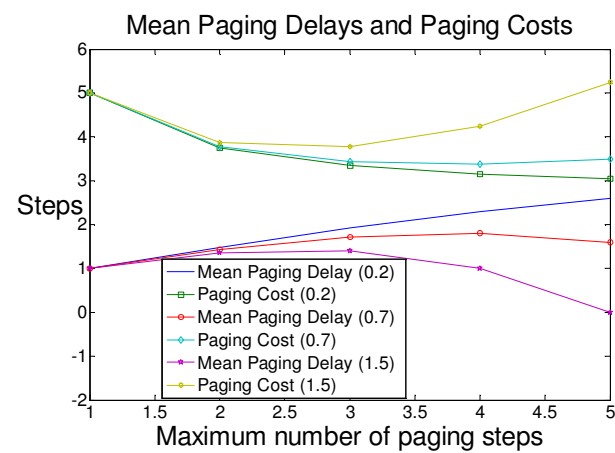

a) Max. steps = 5

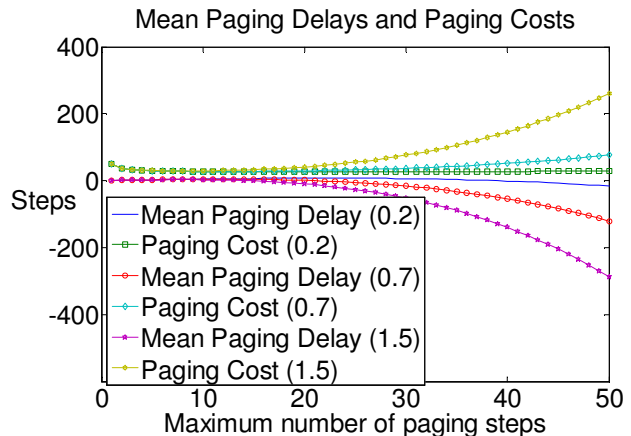

c) Max. steps $=50$

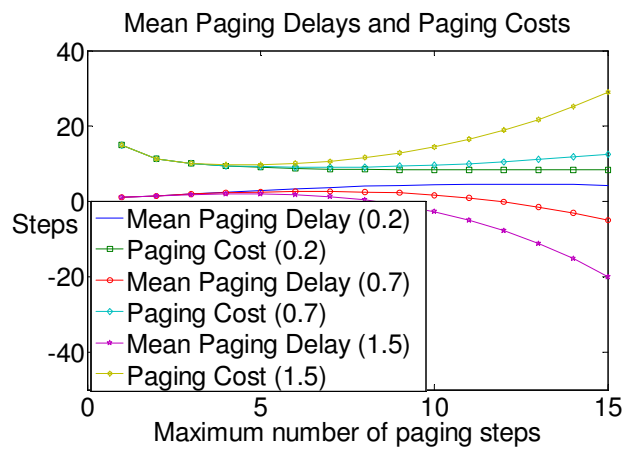

b) Max. steps $=15$

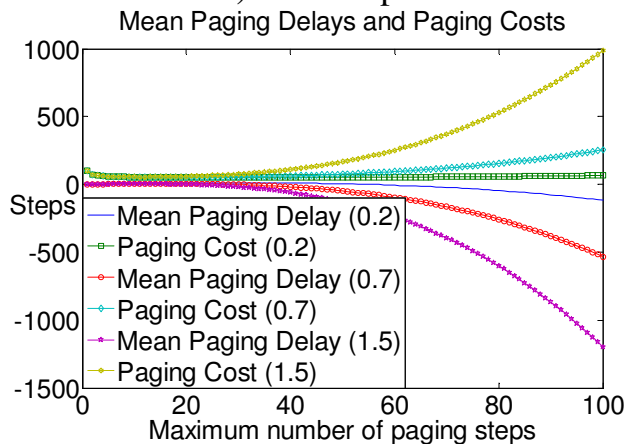

d) Max. steps $=100$

Figure 1. Mean paging delay and paging costs with high values of Lagrange multipliers, for different values of the maximum number of paging steps. 


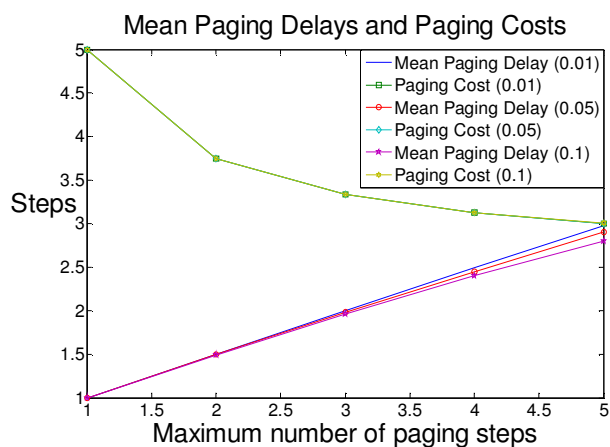

a) Max. steps $=5$

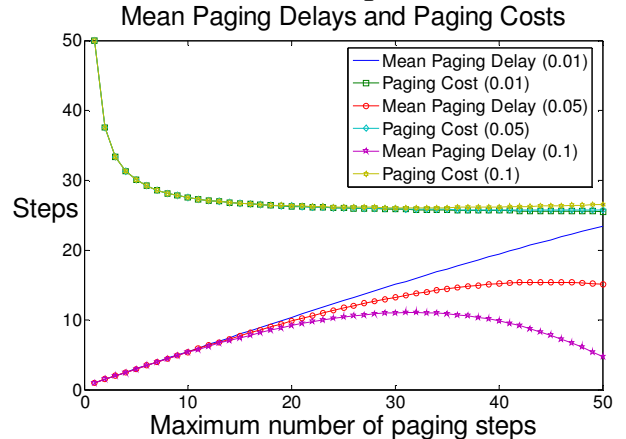

c) Max. steps $=50$

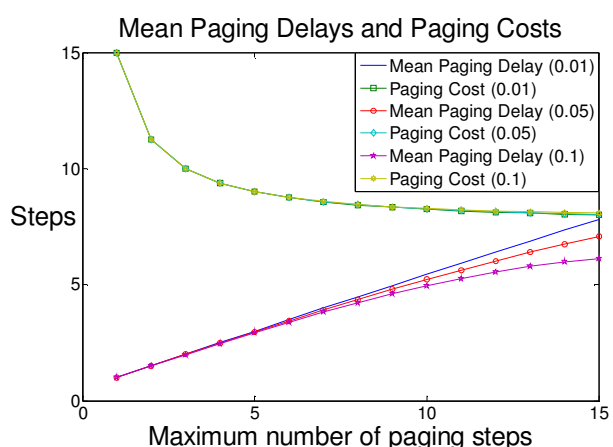

b) Max. steps $=15$

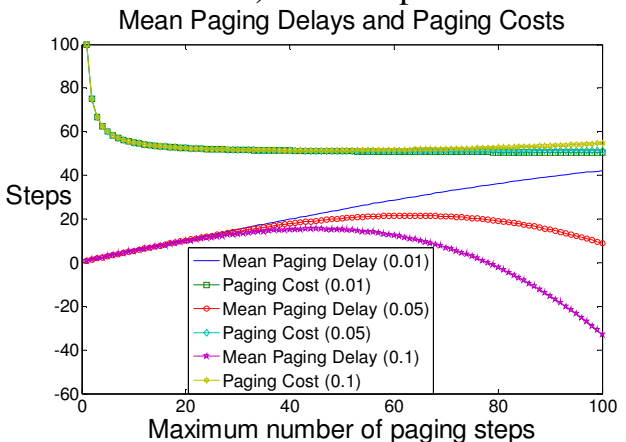

d) Max. steps $=100$

Figure 2. Mean paging delay and paging costs with low values of Lagrange multipliers, for different values of the maximum number of paging steps.

It can be concluded that working with the Lagrange multiplier method to minimize paging costs subject to call delivery delay constraints, in order to obtain proper physical meaning for the behaviour of both magnitudes, the larger the maximum number of paging steps considered, the lower the Lagrange multiplier value (acting as a weighting factor for the paging delay) should be. For instance, as can be observed in Table 2, for a Lagrange multiplier value of 0.1 , the method works properly for a maximum number of paging steps of 32. But as the multiplier value increases to 1.5 or above, the method should be constrained to lower maximum numbers of paging steps, such as 5 or less.

As observed in Figures 1 and 2, the paging costs for lower values of the Lagrange multiplier are nearly identical (upper curves in the figures). And these paging costs vary from the maximum, that is, the total number of locations, for the minimum paging delay (just one paging step), to nearly half of the number of locations, at the expense of higher delays. However, big savings in paging costs $(50 \%)$ can be achieved allowing a delay of, for example, 3 paging steps.

\section{Conclusions}

Having obtained analytical expressions for the Location Management costs by means of the probability distribution for the location of the mobile terminal, their optimisation can be tackled through a wide range of methods.

Applying the classical theory of maxima and minima to minimize the paging costs, we reach the conclusion that whenever the number of cells in the location areas is independent of those location areas probabilities, this theory brings no results. In an attempt to set up a relationship between both terms, considering the total number of cells in the design as a summation of the probabilities of each location area divided by a proportionality constant which measures the 
preference of the designer to give higher priorities to the first locations to be paged, a meaningless solution is obtained again. Further complications of the model do not look worth it, bearing in mind the trade-off between the possible gained accuracy in the solution and the added complexity in the calculations.

Focusing on the minimization of the paging costs subject to the delivery delay as a constraint, the Simplex and the Lagrange Multiplier prove to be suitable methods.

Making use of the Simplex method to the study of deployments of location areas paged sequentially, the following conclusions are drawn:

$>$ When all the probabilities for the location areas are allowed to range freely within the same interval, the optimum solution always implies that the first location area to be paged is imposed the highest possible probability, while the other location areas are assigned the lowest possible probabilities.

$>$ The lower the minimum common bound value for the probabilities of all the location areas, the more the paging costs are reduced. Besides, this same trend is observed for the call delivery delay (used as a constraint), and for the maximum number of required Tableaux, which reflects the computational burden of the Simplex method.

$>$ If a location area different from the first one to be paged is imposed a lower bound constraint for its probability above the rest of location areas, the higher that inferior bound constraint, the larger the paging costs and call delivery delays. And for the same lower bound value, the higher the order number of the location area for which the different constraint is imposed, the larger the paging costs and call delivery delays.

In conclusion, the first location areas to be paged should be assigned superior probabilities in order to minimize paging costs, call delivery delays and even computational costs at the time of applying the Simplex method.

Regarding the application of the Lagrange multiplier method, considering low values of the multiplier, the solutions obtained are coherent with the physical nature of the problem, because the paging costs decline at the expense of a rise in the delay. However, as the maximum number of paging steps grows, the mathematical nature of the method makes the obtained solution lose physical meaning, due to the excessive stress put on minimizing the delay (the more, the higher the multiplier). Consequently, the larger the maximum number of paging steps considered, the lower the Lagrange multiplier value should be. Values of the Lagrange multiplier around 0.1 prove to work efficiently for the typical paging costs optimisation problems and the results obtained show that sequential paging in decreasing order of probability brings large savings in the paging costs with affordable delays: for instance, reduction of $50 \%$ in the costs, for a maximum delay of 3 paging steps. 


\section{REFERENCES}

[1] Martin, E., and Bajcsy, R.; "Savings in Location Management Costs Leveraging User Statistics", International Journal of Ubiquitous Computing, Vol.2, No.3, July 2011, pp. 1-20.

[2] Martin, E., and Bajcsy, R.; "Variability of Location Management Costs with Different Mobilities and Timer Periods to Update Locations", International Journal of Computer Networks \& Communications, Vol.3, No.4, July 2011, pp. 1-15.

[3] Martin, E.; "New Algorithms to Obtain the Different Components of the Location Management Costs", 7th International Conference on Wireless Communications, Networking and Mobile Computing, 2011.

[4] Martin, E.; "Characterization of the Costs provided by the Timer-based method in Location Management", 7th International Conference on Wireless Communications, Networking and Mobile Computing, 2011.

[5] Martin, E.; "A graphical Study of the Timer Based Method for Location Management with the Blocking Probability", 7th International Conference on Wireless Communications, Networking and Mobile Computing, 2011.

[6] Kim, Sung Soo; Kim, Il-Hwan; Mani, V.; Kim, Hyung Jun; Agrawal, Dharma P.; "Partitioning of mobile network into location areas using ant colony optimization", ICIC Express Letters, Part B: Applications, v 1, n 1, p 39-44, September 2010

[7] Almeida-Luz, S. M.; Vega-Rodríguez, M. A.; Gómez-Púlido, J. A.; Sánchez-Pérez, J. M.; "Differential evolution for solving the mobile location management", Applied Soft Computing Journal, v 11, n 1, January 2011, pp. 410-427.

[8] Almeida-Luz, S. M.; Vega-Rodríguez, M. A.; Gómez-Pulido, Juan A.; Sánchez-Pérez, J. M.; "Solving the reporting cells problem using a scatter search based algorithm", Lecture Notes in Computer Science (including subseries Lecture Notes in Artificial Intelligence and Lecture Notes in Bioinformatics), v 6086 LNAI, 2010, pp. 534-543.

[9] Almeida-Luz, S. M.; Vega-Rodríguez, M. A.; Gómez-Pulido, J. A.; Sánchez-Pérez, J. M.; "Applying Scatter Search to the location areas problem", Lecture Notes in Computer Science (including subseries Lecture Notes in Artificial Intelligence and Lecture Notes in Bioinformatics), v 5788 LNCS, 2009, pp. 791-798.

[10] Martin, E., and Bajcsy, R.; "Enhancements in Multimode Localization Accuracy Brought by a Smart Phone-Embedded Magnetometer", IEEE International Conference on Signal Processing Systems, 2011.

[11] Martin, E., and Lin, T.; "Probabilistic Radio Fingerprinting Leveraging SQLite in Smart Phones”, IEEE International Conference on Signal Processing Systems, 2011.

[12] Martin, E.; "Multimode radio fingerprinting for localization", IEEE Radio and Wireless Week, IEEE Topical Conference on Wireless Sensors and Sensor Networks, Phoenix, Arizona, January 2011, pp. 383-386.

[13] Razavi, Sara Modarres; Yuan, Di; Gunnarsson, Fredrik; Moe, Johan; "Optimizing the tradeoff between signaling and reconfiguration: A novel bi-criteria solution approach for revising tracking area design”, IEEE Vehicular Technology Conference, 2009.

[14] Martin, E. and Bajcsy, R.; "Analysis of the Effect of Cognitive Load on Gait with off-the-shelf Accelerometers", Cognitive 2011, Rome, Italy.

[15] Martin, E.; "Solving training issues in the application of the wavelet transform to precisely analyze human body acceleration signals", Proceedings of the 2010 IEEE International Conference on Bioinformatics and Biomedicine, 2010, pp. 427-432.

[16] Martin, E.; "Real time patient's gait monitoring through wireless accelerometers with the wavelet transform", IEEE Radio and Wireless Week, IEEE Topical Conference on Biomedical 
International Journal of Wireless \& Mobile Networks (IJWMN) Vol. 3, No. 4, August 2011

Wireless Technologies, Networks, and Sensing Systems, Phoenix, Arizona, January 2011, pp. 23-26.

[17] Martin, E.; "Novel method for stride length estimation with body area network accelerometers", IEEE Radio and Wireless Week, IEEE Topical Conference on Biomedical Wireless Technologies, Networks, and Sensing Systems, Phoenix, Arizona, January 2011, pp. 79-82.

[18] Martin, E.; “Optimized Gait Analysis Leveraging Wavelet Transform Coefficients from Body Acceleration”, 2011 International Conference on Bioinformatics and Biomedical Technology, March 2011.

[19] Martin, E., and Bajcsy, R.; "Considerations on Time Window Length for the Application of the Wavelet Transform to Analyze Human Body Accelerations”, International Conference Signal Processing Systems, 2011.

[20] Martin, E., Shia, V., Bajcsy, R.; "Determination of a Patient's Speed and Stride Length Minimizing Hardware Requirements”, Body Sensor Networks, May 2011.

[21] Martin, E. et al., "Linking Computer Vision with off-the-shelf Accelerometry through Kinetic Energy for Precise Localization”, International Conference Semantic Computing, 2011.

[22] Martin, E. et al., "Enhancing Context Awareness with Activity Recognition and Radio Fingerprinting", International Conference Semantic Computing, 2011.

[23] Hancock, H., "Theory of Maxima and Minima”. Dover Publications, New York, 1960.

[24] Hildebrand, F. B., "Introduction to Numerical Analysis". McGraw-Hill, New York, 1956.

[25] Ralston, A., “A First Course in Numerical Analysis”. McGraw-Hill, New York, 1965.

[26] Helou, A., J., "Some aspects of optimisation", MSc Dissertation, 1976, School of Chemical Engineering, University of Bradford, UK.

[27] Dantzig, G. B., "Linear Programming and Extensions". Princeton, New Jersey: Princeton University Press, 1963.

[28] Dantzig, G. B., "Expected number of steps of the Simplex method for a linear program with a convexity constraint", Technical report SOL 80-3, 1980, Standford University

[29] Klee, V., and Minty G. J., "How good is the Simplex algorithm?" Inequalities III, 1972, O. Shisha, ed. New York: Academic Press, p. 159-175.

[30] Rose, C., and Yates, R., "Minimizing the average cost of paging under delay constraints". ACM wireless Networks, 1997, v1, n2, p. 211-219.

\section{Authors}

E. Martin is carrying out research in the Department of Electrical Engineering and Computer Science at University of California, Berkeley. He holds a MS in Telecommunications Engineering from Spain and a $\mathrm{PhD}$ from England within the field of location management for mobile telecommunications networks. He has research experience in both industry and academia across Europe and USA, focusing on wireless communications, sensor networks, signal processing and localization.

R. Bajesy received the Master's and Ph.D. degrees in electrical engineering from the Slovak Republic, and the Ph.D. in computer science from Stanford University, California. She is a Professor of Electrical Engineering and Computer Sciences at the University of California, Berkeley. Prior to joining Berkeley, she headed the Computer and Information Science and Engineering Directorate at the National Science Foundation. Dr. Bajcsy is a member of the National Academy of Engineering and the National Academy of Science Institute of Medicine as well as a Fellow of the Association for Computing Machinery (ACM) and the American Association for Artificial Intelligence. In 2001, she received the ACM/Association for the Advancement of Artificial Intelligence Allen Newell Award, and was named as one of the 50 most important women in science in the November 2002 issue of Discover Magazine. 